\title{
Design on Micro Video English Teaching Resources Platform Peng Zhang ${ }^{a}$, Xiuli Gou ${ }^{b}$ \\ College of Foreign Languages, Bohai University, Jinzhou, 121013, China \\ azhangpeng44444@126.com, b1029915835@qq.com
}

Keywords: micro video; English teaching resources; platform design; video coding; database design; technology framework

\begin{abstract}
Micro video resources applied in English teaching, English teaching conforms to the development direction of modern education technology. In order to promote the micro video teaching resources in English teaching, the application of resource management platform is designed. Design content includes four parts: first, technical framework, using the struts 2, stage play using a large number of interceptors and the advantages of the various views support; The second is, database, logical structure design based on Oracle database management system; Three is that video coding, based on the H. 264 coding standard, improve the coding efficiency, flexibility, and customization features; Four is that the server implementation process, consisting of core services and processing module, and design the interface between the two. In this paper, the research results, for the construction for personalized micro video English teaching resources platform provides the basis.
\end{abstract}

\section{Introduction}

Micro video refers to the short 30 seconds, long is no more than 20 minutes of video clips. Micro video content is broader, video form is diversiform, usually covers the micro film, record video, DV video, video clips and advertising clips, etc. Micro video by PC, mobile phone, camera, DV, DC and MP4 and other video terminal video recording or playback. "Short, fast, pure", public participation and optional sex is the biggest characteristic of micro video anytime and anywhere. The classroom teaching based on micro video teaching resources can make the study content of this subject teaches becomes vivid, image, intuitive, cause the attention of learners to the learning content, to improve learners' learning enthusiasm [1]. Micro video resources applied in English teaching, English teaching conforms to the development direction of modern education technology. Teaching resources provide the necessary conditions for the excellent teaching resources sharing platform, for teachers' teaching, students' autonomous learning resources provide effective teaching interaction. The development and application of micro video teaching resources, has been the hot topic in education technology. Based on micro video English teaching resources is difficult to the present situation of the management and application, the design resource management platform, to provide solution for system development, further promote the micro video teaching resources application in English teaching.

\section{Technology Framework Design}

Struts 2 is the Struts of the next generation products, are in the Struts 2 and WebWork technology based on the new framework of the merger. Struts 2 WebWork as the core, using interceptor mechanism to deal with the user's request, make business logic controller and ServletAPI completely away from [2, 3]. Struts 2 is to provide a large number of interceptors, based on plug-in framework, multiple view support, more modular, and Spring integration, based on the advantages of pojo is easy to test, so more and more widely used. It is because of the powerful features of the struts 2 and advantages, in software development for a wide range of applications.

The Struts2 framework can be roughly divided into three parts: core controller FilterDispatcher users, business controller Action, and implement business logic components. Among them, the core controller FilterDispatcher is the basis of the Struts 2 framework, includes a framework of internal 
control process and the processing mechanism. Business controller Action and the business logic component need the user to their implementation. Users in the development of the Action and the business logic components at the same time, also need to write the relevant configuration files, for core controller FilterDispatcher to use; Business controller Action is write your own implementation by the developer, the Action class can be a simple Java classes, and the separation from the Servlet API. Action usually have an execute () method, which can also define other business control method; Business model components can be implement the business logic module, can be the EJB, POJO or JavaBean, in actual development, to the distinction between the business model components and definitions are vague, is beyond the scope of the Struts 2 framework. Different developers or teams, have their own way to implement the business logic module, the purpose of the Struts 2 framework is to use Action to invoke the business logic module. The Struts2 framework, the structure is shown in Fig. 1.

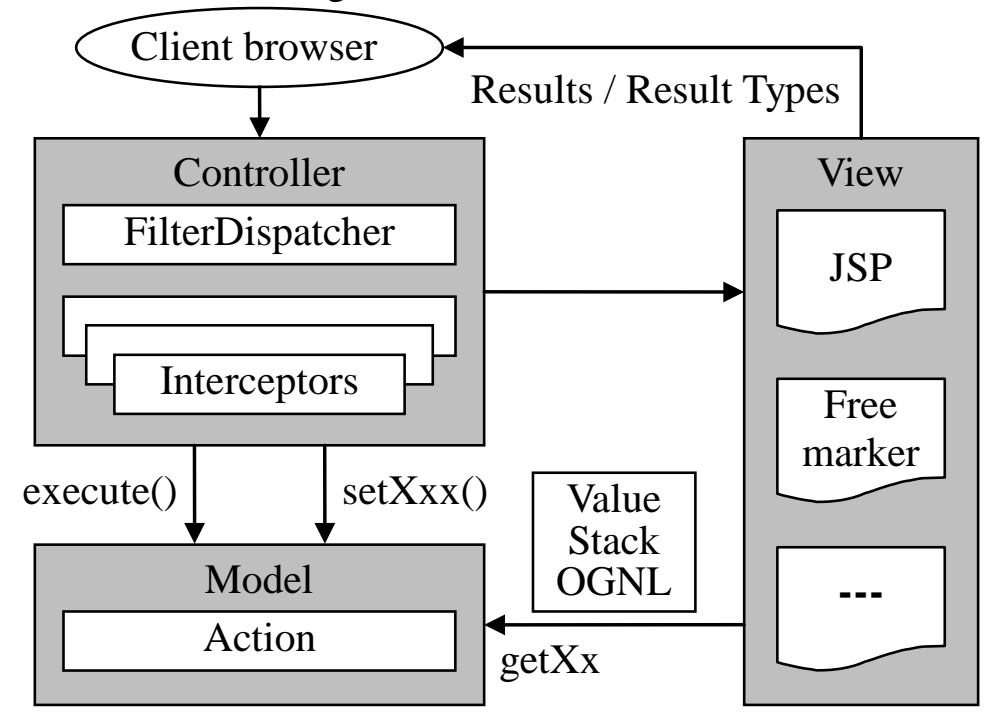

Fig. 1. Technology framework of Struts2

\section{Database Design}

Database design is to establish a database and its application technology in the system, is an important part of the software design. Specifically, the database design is for a given application environment, to construct the optimal database schema, to establish database and application system, to store data effectively and meet the application requirements of various users.

Micro video database design is the purpose of English teaching resources for teachers and students to provide powerful information data retrieval, processing and query function, which improve the efficiency of teachers' work and the students' English level. Database design should follow the principle of sharing in the process, the principle of integrity, security, scalability, maintainability and standardization principle, the principle of sharing refers to coordinate various data sources, do algorithm is unified, consistent measurement; Integrity principle is index, according to the validity and compatibility of the input data for review and constraint mechanism; Safety principle is refers to protect the data; Scalability principles mainly database upgrade the scalability of the job; Standardization principles for data storage should follow the standard theory. Usually the database design includes concept structure design, logical structure and physical structure design. The limit of paper length, only logical structure design results are given in this paper. Based on the logical structure of Oracle database management system design results are shown in Table 1.

The communist party of China including 6 types of data in Table 1, the following brief description [4] : the ROWID, detailed address, in which data will be stored by the ROWID, Oracle can quickly locate the position of a line of specific data; CHAR, storage fixed-length string, if the input field values less than the limit length of field, actually stored data, automatically complement 
Spaces to the right before, to the content of the field values stored in the data block; VARCHAR2, stored longer string, when the input field values less than the limit length of the field, directly to the content of the field values stored in the data block, rather than fill the gaps, save the data block space; NUMBER, storage precision up to 38 Numbers, each NUMBER stored in a variable length field, length between $0 \sim 22$ bytes; The DATE, the DATE is used to store data, although can be expressed in characters or digital type DATE information, but the DATE data type associated with special properties; CLOB, large-scale storage and unstructured data, including text, images, video, and data storage space, etc., to support a fixed width and variable width of the character set.

Table 1. Logical Structure Design on micro video English teaching resources platform

\begin{tabular}{clcc|clcc}
\hline No & Fields Name & Type & Bytes & No & Fields Name & Type & Bytes \\
\hline 1 & ResourceCoding & ROWID & & 12 & AttachAmount & NUMBER & 4,0 \\
2 & TypeCoding & CHAR & 4 & 3 & BrowsingTimes & NUMBER & 10,0 \\
3 & SourceCoding & CHAR & 4 & 14 & DownloadTimes & NUMBER & 10,0 \\
4 & ApplyCoding & CHAR & 4 & 15 & StorageAddress & VARCHAR2 & 500 \\
5 & StaffCoding & CHAR & 4 & 16 & UploadDate & DATE & \\
6 & GradedCoding & CHAR & 4 & 17 & UploadTime & DATE & \\
7 & FormatCoding & CHAR & 4 & 18 & UploadIP & CHAR & 15 \\
8 & AuthorityCoding & CHAR & 4 & 19 & ResourceEvaluation & VARCHAR2 & 500 \\
9 & AuthorCoding & CHAR & 4 & 20 & UseEnvironment & VARCHAR2 & 500 \\
10 & ResourceName & VARCHAR2 & 50 & 21 & Instructions & CLOB & \\
11 & ResourceSize & NUMBER & 6,2 & 22 & ResourceProfile & CLOB & \\
\hline
\end{tabular}

\section{Video Coding Technology}

Video coding is to point to by specific compression technology to converts a video format file to another format video file. Video streaming transmission of the most important coding standards have ITU (International Telecommunication Union) of h. 261, h. 263, h. 264, movement of static image expert group M - a JPEG of the MPEG series standards and the International organization for standardization the moving pictures experts group, is widely used on the Internet and Real Networks RealVideo, Microsoft's Windows media and Apple's QuickTime, etc. This platform using coding standard h. 264, coding structure from the function and the algorithm is divided into two layers, namely, video coding and network abstraction layer, as shown in Fig. 2.

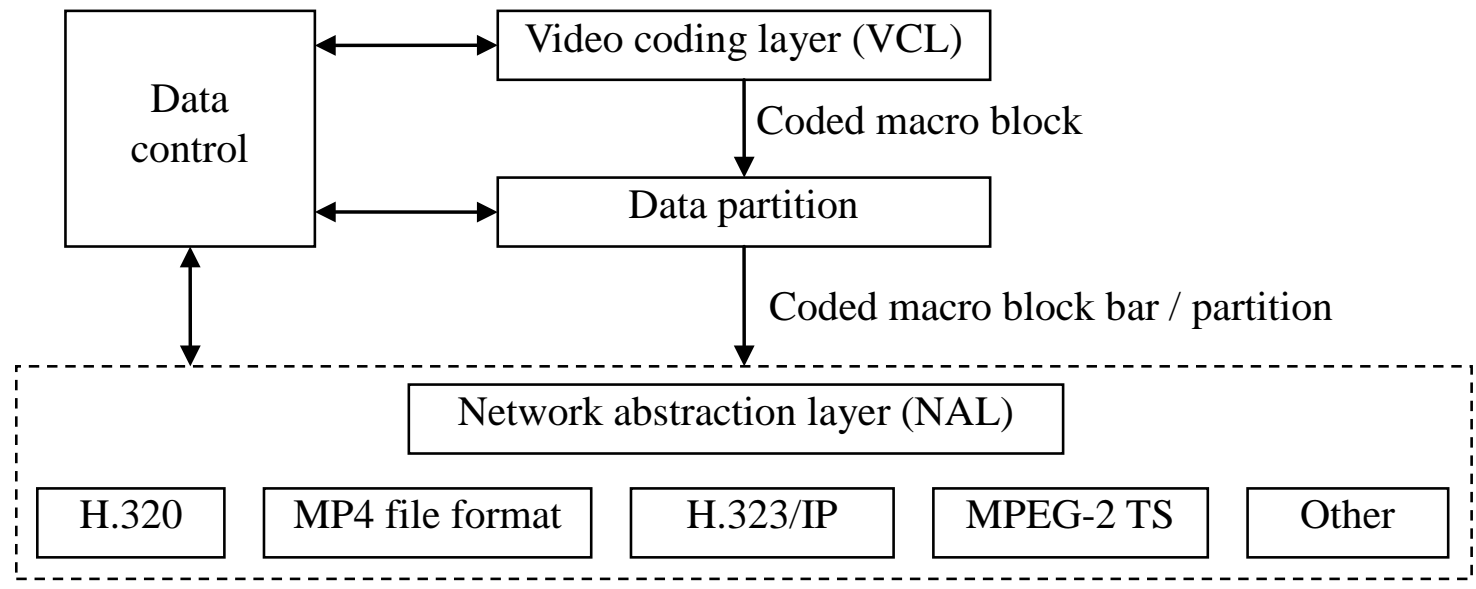

Fig. 2. Layered structure of H.264

The VCL is responsible for the efficient video coding compression, using block-based motion compensation prediction, transform coding and entropy coding hybrid coding framework, combining the processing object is block and macro block of data. VCL is the core of video coding, which contains many implementation error recovery tool, and mining has a lot of advanced 
technology to improve the coding efficiency and video coding [5]; NAL will go through the VCL layer coded video stream segmentation and packaging, further provide adaptive ability to different network performance matching is responsible for the network adapter. NAL layer in the NAL unit as the basic data format, not only contains all video information, also provides the transport layer and storage media information, in the transmission of packet switching can eliminate framing and heavy synchronization overhead.

H. 264 is the ISO (International Organization for Standardization) and (ITU), is a new generation of digital after MPEG4 video compression format, which retain the advantages of the traditional compression techniques and essence, but also has many advantages unmatched by other compression techniques [6, 7] : Low Bit Rate, and MPEG2 and MPEG4 compression technology such as ASP, compared under the same image quality, H. 264 technology compressed data volume is only about 1/8 of the MPEG2, 1/3 of the MPEG4. H. 264 compression technology using save users download time and data traffic; High quality images, H. 264 can provide continuous and smooth high quality images; Fault tolerant ability, H. 264 provides solve the unstable network environment prone to error of necessary tools such as packet loss; Network, strong adaptability, $\mathrm{H}$. 264 provides network adaptation layer, makes the h. 264 file can easily on the different network transmission.

\section{Server Implementation Process}

Server implementation by the Core Service (Core Service) and processing module (Handle) Modules of two parts, a Core Service is the interface between the client and processing module, the client through the RTP and RTSP request sending and receiving the response, processing module is responsible for processing requests and transmit packets to the client. The implementation process is shown in Fig. 3.

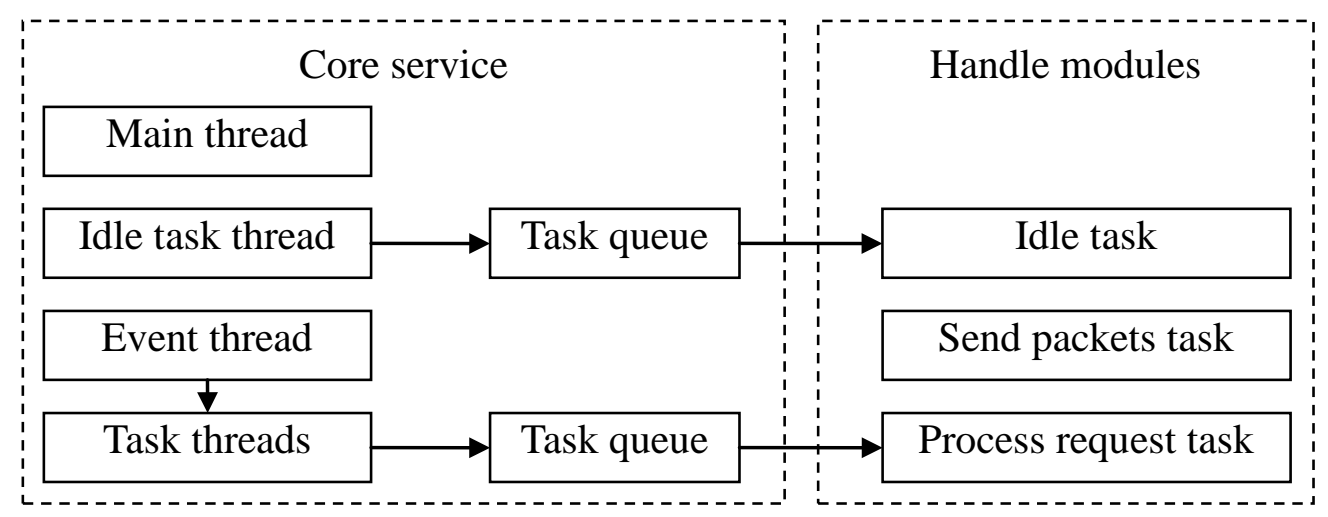

Fig. 3. Implementation process on streaming media server

For streaming media server as shown in Fig. 3 implementation process description is as follows: the Main Thread (the Main Thread) is responsible for check the server status and control input and output; Idle Task threads (Idle Task Thread) management of periodic Task Queue, including overtime tasks and set of interface Task, the Task Queue (Task Queue), the Task Queue is sent to the processing module of Idle Task (Idle Task) module for processing; The Event Thread (the Event Thread) is responsible for receiving and RTP, RTSP request packet, and transmitted to the task threads. Task Threads (Task Threads), there are usually multiple Task Process, from the event thread receive RTSP Request and RTP packets are placed in the Task queue, send Task queue to the processing module processes the Request Task (Process Request Task) module for processing. Processing module to Send Task (Send Packets Task) module will process request packet is sent to the client.

Task object using two methods, responsible for Signal sends an event Task object, can be sent to a process or a set of processes of short message, if the same event is sent multiple times, only one will be into the accepting process of suspension queue; Run for the Task object specifies the processing time, when there are events need to be addressed when called, can call GetEvents 
function receives the current and past has used signal through the event, and automatically make the events from the queue.

\section{Conclusion}

Information technology has a revolutionary influence on education development, must be highly valued. The use of information technology promotes the education informatization, drive the modernization of education and the innovation and reform of education. Micro video as a kind of brand-new teaching resources has the teaching time is short, vivid, and the advantages of the fragmentation of the complex course content, adapted to the needs of English teaching. The traditional teaching of the three elements of teaching content, teachers and students, teaching resources just as resources "warehouse", only to supplement of classroom teaching [8]. The development of information technology, the teaching content of learning is not only in classroom, students can access networks, using a variety of teaching resources for learning. In this paper, through the use of advanced education of information technology, the construction for personalized video English teaching resources platform, for teachers and students easily select, acquire, using and management of resources to provide quality service, realize the personalized learning, promote the co-construction and sharing of teaching resources.

\section{Acknowledgement}

This work is supported by social science fund project of Liaoning province: Construction and application on micro video English teaching resources platform (L14DYY029).

\section{References}

[1] Md. Mahedi Hassan, Poo Kuan Hoong, "Seamless handover integrated solution for video transmission over proxy mobile IPv6 in a micro mobility domain," Journal of Network and Computer Applications, vol. 36, no. 1, pp. 66-76, 2013.

[2] Z. G. Zhang, "Development and application on Struts2 framework technology," Computer CD Software and Applications, vol. 16, no. 23, pp. 119-120, 2013.

[3] X. B. Chen, "Advantages of struts2," Dhttp://xiaobing259-163-com.iteye.com/blog/1530960, 2016-9-18.

[4] Xiaoxiang Hermit, "Summary of ORACLE basic data types," http://www.cnblogs.com/kerrycode/archive/2013/08/17/3265120.html, 2016-9-19.

[5] J. F. Ren, J. Yao, L. Guo, "H.264/AVC and Its Application in IP Network," Computer Engineering, vol. 31, no. 17, pp. 105-107, 2005.

[6] Blog Garden, "H.264 profile," http://kb.cnblogs.com/page/168157/, 2016-9-18.

[7] J. Dai, S. L. Zhang, Q. J. Li, Y. J. Ma, "On the key technology of H.264 video coding standard," Digital Technology and Application, vol. 34, no. 7, pp. 233-233, 2016.

[8] S. Yang, "Design and Implementation of Resource Platform for Individualized Instruction," Master's Degree of Huazhong Normal University, 2015. 